\title{
Entropy Generation in Rayleigh-Bénard Problem for Different Geometrical Cavities
}

\author{
Omar Al-Abbasi \\ Department of Mechanical Engineering, College of Engineering, University of Bahrain \\ omabbasi@uob.edu.bh
}

Received 17 May 2017, Accepted 16 October 2017

\begin{abstract}
Entropy generation for the natural convection phenomena is studied for different geometrical cavities that have identical boundary conditions and unit area. The key characteristic parameter in this study was the Grashof number. The effect of Grashof number on Nusselt number and entropy generation has been studied using finite element method. The developed models in this study were validated against published work in the literature and results were found to be in close agreement. Entropy generation was found to increase with an increase in the Grashof number and the heat transfer was the dominant factor in increasing the entropy generation. A lower entropy generation is generally expected with an increase in the distance between the hot and cold boundaries. It is also noted that the rate of entropy generation decreases slowly when the flow domain is only slightly distorted, while the rate of entropy generation increases sharply when the flow domain changes significantly.
\end{abstract}

Keywords: Rayleigh-Bénard; entropy generation; heat transfer.

\section{Introduction}

The natural convection phenomenon plays an important role in several engineering applications, among few which are the cooling process of electronic circuit boards, solar collectors, thermal storage, and environmental comfort. Natural convection, caused by temperature differences, is a key reason for large scale air circulation in the atmosphere. The temperature gradient affects the density which in turn creates the buoyancy force that drives the flow domain.

The literature is rich with a lot of studies investigating several aspects of Benard problem in enclosed cavities and some of that work has been devoted to investigate the entropy generation phenomenon. Brahim et al. [1] investigated the entropy generation inside a square cavity at the onset of natural convection. Entropy generation in a square cavity as a function of the position of heat sources located on top and bottom walls has been investigated by Prakash et al. [2]. On the other hand, Hooman and Fmouri [3] studied the entropy generation process when the heated element is located at different positions inside the square cavity. Basak et al. [4] studied the effect of the inclination angle on the entropy generation for a square cavity. In an earlier study, Baytas [5] investigated the optimum inclination that minimizes the entropy generation in a square cavity. Another geometrical aspect which is the aspect ratio and its effect on entropy generation in a rectangular cavity has been studied by Oliveski et al. [6] and Sunden et al. [7]. For triangular enclosure, Varol et al. $[8,9]$ studied the entropy generation for isosceles triangle partially heated from below and the effect of the inclination angle with a non-uniformly heated boundary. For cylindrical geometry, Abu-Hijleh et al. [10] investigated the entropy generation for a horizontal cylinder and Abu-Hijleh and Heilen [11] investigated the phenomena for a rotating cylinder. For other complex geometry, Aounallah et al. [12] studied entropy generation in an enclosure with a wavy hot wall. A more thorough literature review can be found in the work of Oztop and Al-Salem [13].

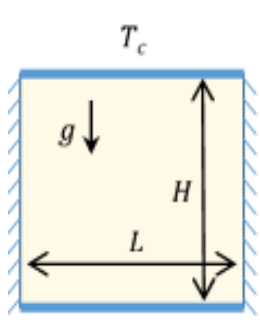

$T_{h}$

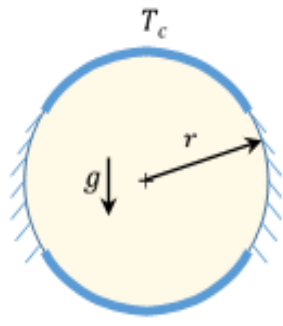

$T_{h}$

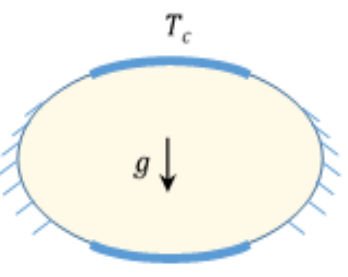

$T_{h}$

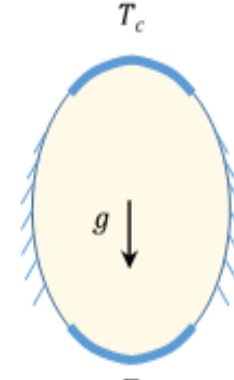

$T_{h}$

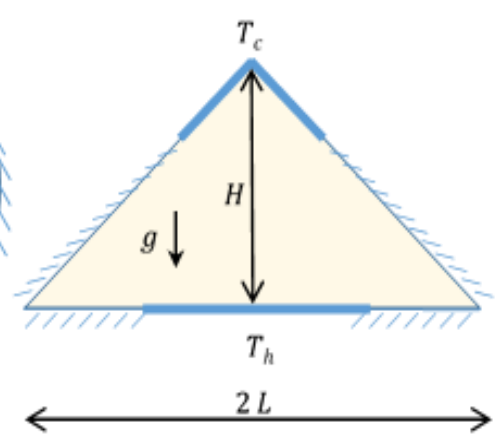

Figure 1. The different configuration in this study (hot and cold boundaries are shown in bold lines). 
The aim of the current work is to investigate how the geometry is affecting the production of entropy in the natural convection process of Rayleigh-Benard problem. Several geometries are investigated in this work, i.e., square, circle, horizontal and vertical ellipse and triangle as shown in Figure 1. All these geometries have a unit area with hot and cold boundaries of unit length, as depicted in the figure.

\section{Mathematical Model}

Five different cavities are considered in this study. The shapes include square, circle, vertical ellipse, horizontal ellipse and triangle as shown in Figure 1. In order to study the effect of the shape on entropy generation, all configurations had the same area. Moreover, the length of the boundaries where the temperatures are prescribed was also kept constant.

The time-independent governing equations for the conservation of mass, momentum and energy are given respectively by:

$\nabla(\rho u)=0$

$(\rho \boldsymbol{u} \cdot \nabla) \boldsymbol{u}=-\nabla p+\mu \nabla^{2} \boldsymbol{u}+\rho g \beta\left(T-T_{c}\right) \hat{\jmath}$

$(\boldsymbol{u} \cdot \nabla) T=\alpha \nabla^{2} T$

where $\boldsymbol{u}$ is the velocity vector in both $\mathrm{x}$ and y directions, $\rho$ the density, $\mathrm{p}$ the pressure, $\mathrm{T}$ the temperature and $T_{c}$ the cold wall temperature, $\mu, \beta$ and $\alpha$ are the fluid absolute viscosity, thermal expansion and thermal diffusivity, respectively, $g$ the gravitation constant and $\hat{\jmath}$ is the unit vector in the $\mathrm{y}$ direction. The fluid properties are assumed to be constant.

The local entropy generation due to heat transfer and fluid friction is given according to the following expression:

$s=\frac{k}{T^{2}}(\nabla T)^{2}+\frac{\mu}{T} \Phi$

where $k$ is the thermal conductivity, $\Phi$ the viscous dissipation function that can be given in two- dimensional Cartesian coordinates by:

$\Phi=\frac{\mu}{T}\left\{2\left[\left(\frac{\partial u}{\partial y}\right)^{2}+\left(\frac{\partial v}{\partial x}\right)^{2}\right]+\left(\frac{\partial u}{\partial y}+\frac{\partial v}{\partial x}\right)^{2}\right\}$

where $u$ and $v$ are the velocity components in $x$ and $y$ directions, respectively.

In order to write Eqs. (1) - (3) in a non-dimensional form, the following non-dimensional parameters have been used

$\hat{x}=\frac{x}{L^{2}}, \hat{y}=\frac{y}{L^{x}}, \hat{u}=\frac{u}{\sqrt{g \beta \Delta T L}} x \hat{v}=\frac{v}{\sqrt{g \beta \Delta T L}} x^{x}=\frac{p}{\rho g \beta \Delta T L}$,

$\theta=\frac{T-T_{c}}{T_{h}-T_{c}}, P r=\frac{v}{\alpha}, G r=\frac{g \beta L^{3}\left(T_{h}-T_{c}\right)}{v^{2}}$

where $\hat{u}$ and $\hat{v}$ are the dimensionless velocities in $\mathrm{x}$ and $y$ directions, respectively, $\theta$ is the dimensionless temperature, $v$ the kinematic viscosity, and Pr and Gr the Prandtl and
Grashof numbers, respectively. Upon using these dimensionless parameters in Eqs. (1) - (3), the governing equations can be written as follows

$\nabla \hat{u}=0$

$(\hat{u} \cdot \nabla) \hat{u}=-\nabla \hat{p}+\frac{1}{\sqrt{G r}} \nabla^{2} \hat{u}+\theta \hat{\jmath}$

$(\widehat{u} \cdot \nabla) \theta=\frac{1}{\operatorname{Pr} \sqrt{G r}} \nabla^{2} \theta$

Similarly, the non-dimensional entropy generation rate per unit area, i.e., Eq.(4), is transformed using the above dimensionless parameters in a two-dimensional Cartesian coordinates as follows

$$
\begin{aligned}
& \hat{s}=\frac{1}{(\theta+\tau)^{2}}\left\{\left(\frac{\partial \theta}{\partial \hat{x}}\right)^{2}+\left(\frac{\partial \theta}{\partial \hat{y}}\right)^{2}\right\}+ \\
& \left(\frac{1}{\theta+\tau}\right) \frac{E c}{\operatorname{Pr}}\left\{2\left[\left(\frac{\partial \hat{u}}{\partial \hat{y}}\right)^{2}+\left(\frac{\partial \hat{v}}{\partial \hat{x}}\right)^{2}\right]+\left(\frac{\partial \hat{u}}{\partial \hat{y}}+\frac{\partial \hat{v}}{\partial \hat{x}}\right)^{2}\right\}
\end{aligned}
$$

where $E c$ is the Eckert number given by $\mathrm{u}^{2} /\left(\mathrm{c}_{\mathrm{P}} \mathrm{T}\right)$ and $\tau$ the dimensionless temperature difference given by $T_{c} /\left(T_{h}-T_{c}\right)$ and the total entropy generation rate is estimated by integrating Eq.(10) over the entire domain

$\widehat{S_{t}}=\int_{A} \widehat{S^{2}} d A$

\section{Model Validation}

Several researchers 1, 3, 4, 6 studied this problem numerically where Rayleigh number ( $\mathrm{Ra}$ ) was the governing parameter describing the flow. However, based on the choice of the dimensionless parameters, the Grashof number $(\mathrm{Gr})$ is the parameter that described the flow in this study. Also, different scaling parameters has been used to nondimensionalize the governing equations. In the previously mentioned studies, the governing equations are given as

$$
\nabla \tilde{u}=0
$$

$$
(\tilde{u} \cdot \nabla) \tilde{u}=-\nabla \hat{p}+\operatorname{Pr} \nabla^{2} \tilde{u}+\operatorname{Ra} \operatorname{Pr} \theta \hat{j}
$$

$$
(\tilde{u} \cdot \nabla) \theta=\nabla^{2} \theta
$$

and the local entropy generation is given by:

$$
\begin{aligned}
& \xi=\left\{\left(\frac{\partial \theta}{\partial \tilde{x}}\right)^{2}+\left(\frac{\partial \theta}{\partial \tilde{y}}\right)^{2}\right\}+ \\
& \phi\left\{2\left[\left(\frac{\partial \tilde{u}}{\partial \tilde{y}}\right)^{2}+\left(\frac{\partial \tilde{v}}{\partial \tilde{x}}\right)^{2}\right]+\left(\frac{\partial \tilde{u}}{\partial \tilde{y}}+\frac{\partial \tilde{v}}{\partial \tilde{x}}\right)^{2}\right\}
\end{aligned}
$$

where $\varnothing=\frac{\mu T_{0}}{k}\left(\frac{\alpha}{(L \Delta T)^{2}}\right)$, which is defined as the irreversibility. 
In Eqs. (12) - (15), $\tilde{u}$ is the flow field that is driven by different set of dimensionless parameters, i.e., $\tilde{u}=(L / \alpha) u$ . In order to validate the results of this study with those existing in the literature, a simple transformation between the two flow fields, i.e., $\widehat{\boldsymbol{u}}$ and $\tilde{\boldsymbol{u}}$ was done and the flow fields were related by

$$
\tilde{\boldsymbol{u}}=\sqrt{G r} \operatorname{Pr} \hat{\boldsymbol{u}}
$$

In order to calculate the entropy generation by Eq. (15), the flow field given by the solution of the governing equations (7) - (9) had to be transformed using Eq. (16) The benchmark model is depicted in Figure 2. Two parameters were investigated in this phase, i.e., the entropy generation given by Eq. (15) and Nusselt number $(\mathrm{Nu})$.

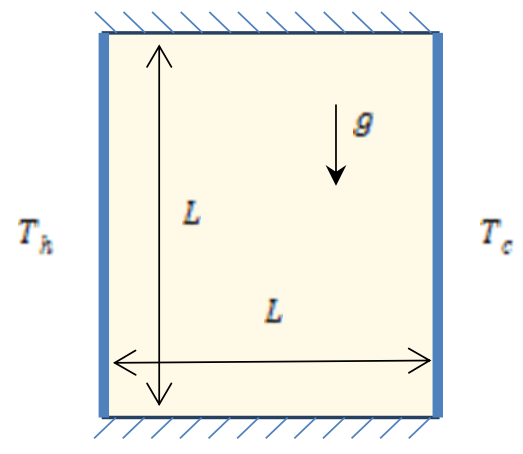

Figure 2. The benchmark model.

The dimensionless entropy generation predicted by the current model was benchmarked with data existing in the literature, as shown in Figure 3, where the irreversibility parameter $\Phi$ is 0.01 . It shows a good agreement between the entropy generation predicted by this study and the literature.

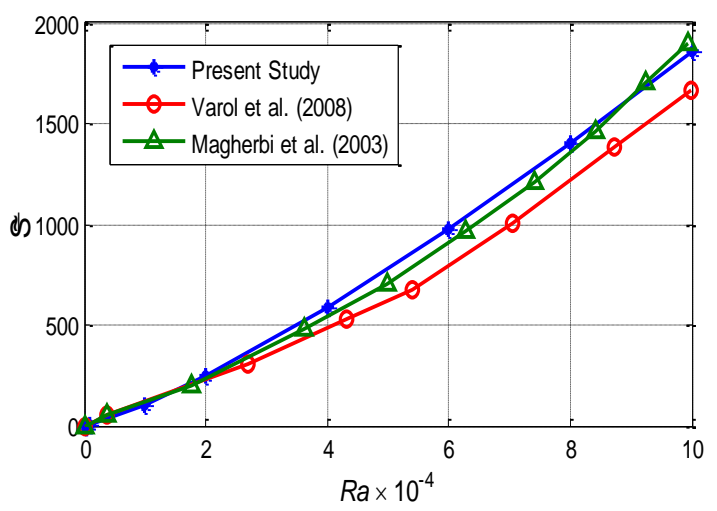

Figure 3. A comparison of the dimensionless entopy generation with data in the literature.

The local Nusselt number $(\mathrm{Nu})$ is given by the following expression

$N u=\left(-\frac{\partial \theta}{\partial n}\right)_{\text {hotwall }}$

where $n$ is the unit vector normal to the plane, and the mean Nusselt number (Num) is given by
$N u_{m}=\frac{1}{L} \int_{0}^{L} N u d s$.

The obtained values of Num were validated against two studies, Oliverski 6 and Davis et al. 14 as shown in Table 1 and the results showed that the current model is in good agreement with the results of these studies.

Table 1. Benchmarking Nusselt number vs. Rayleigh number.

\begin{tabular}{l|c|c|c|c|} 
& \multicolumn{4}{c}{$N u_{m}$} \\
\cline { 2 - 5 } & $R a=10^{7} R a=10^{4}$ & $R a=10^{5}$ & $R a=10^{6}$ \\
\hline Oliveski et al. ${ }^{6}$ & 1.116 & 2.239 & 4.531 & 8.726 \\
\hline Davis et al. ${ }^{14}$ & 1.118 & 2.243 & 4.519 & 8.800 \\
\hline Present study & 1.1176 & 2.2462 & 4.5192 & 8.8326 \\
\hline
\end{tabular}

\section{Numerical Procedure}

The governing equations were solved using COMSOL Multiphysics software. The momentum and energy equations were solved using PARDISO direct solver. The solution relative tolerance was set to $1 \times 10^{-4}$. The $\hat{u}, \hat{p}$ and $\theta$ have been formulated using linear shape functions. The domain was discretized using free triangular elements and rectangular elements were used on the boundaries to ensure capturing of the boundary layer for the flow field. This is shown by a coarse mesh depicted by Figure 4 .

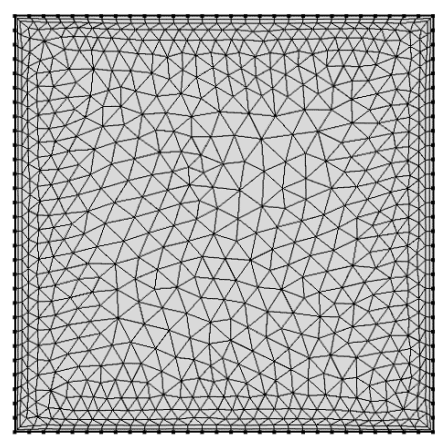

Figure 4. Depiction of a coarse mesh of the domain.

\section{Results and Discussion}

In this study, the Prandtl number $(P r)$ was fixed to 0.7 and Eckert number $(E c)$ that appears in Eq. (10) is expressed as

$E c=\frac{u^{2}}{c_{D} \Delta T}$.

Using the non-dimensional parameters of Eq.(6), this number is transformed to

$E c=\frac{g \beta L}{C_{p}} \widehat{u}^{2}$

where $C_{p}$ is the specific heat at a constant pressure and was set as $1.005[\mathrm{~kJ} /(\mathrm{kg} \mathrm{K})], \beta$ equals $3.3 \times 10^{-3}[1 / \mathrm{K}], L$ is $1[\mathrm{~m}]$ and $\hat{\mathrm{u}}$ is the solution obtained from solving the governing Eqs. (7) - (9). To estimate the local non-dimensional entropy generation given by Eq. (10), the dimensionless 
Table 2. Grid independency test for the total entropy generation $\widehat{\mathrm{S}_{\mathrm{t}}}$ at $G r=1000$.

\begin{tabular}{|c|c|c|c|c|c}
\hline \multirow{2}{*}{ Shape } & \multirow{2}{*}{$\begin{array}{c}\text { Mesh 1 } \\
\text { \# of elements }\end{array}$} & $\begin{array}{c}\text { Mesh 2 } \\
\text { \# of elements }\end{array}$ & \multicolumn{2}{|c|}{$\hat{S}_{t}$} & \multirow{2}{*}{ Relative error \% } \\
\cline { 3 - 5 } & & 15850 & 0.010186 & 0.010205 & 0.1862 \\
\hline Square & 5998 & 6486 & 0.013190 & 0.013259 & 0.5204 \\
\hline Circle & 3688 & 3418 & 0.021377 & 0.021394 & 0.0795 \\
\hline Hellipse & 2038 & 9128 & 0.012052 & 0.012027 & 0.2079 \\
\hline Vellipse & 3668 & 17780 & 0.017170 & 0.017179 & 0.0524 \\
\hline Triangle & 7214 & & & & \\
\hline
\end{tabular}

a
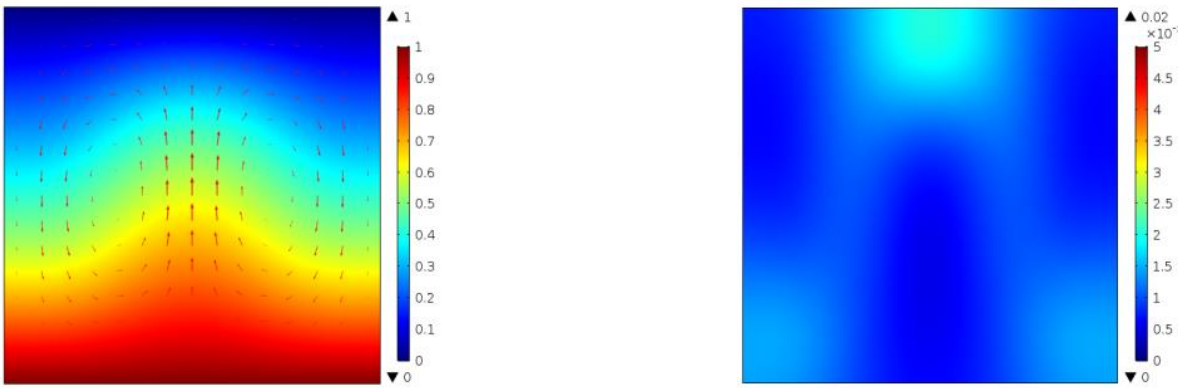

b
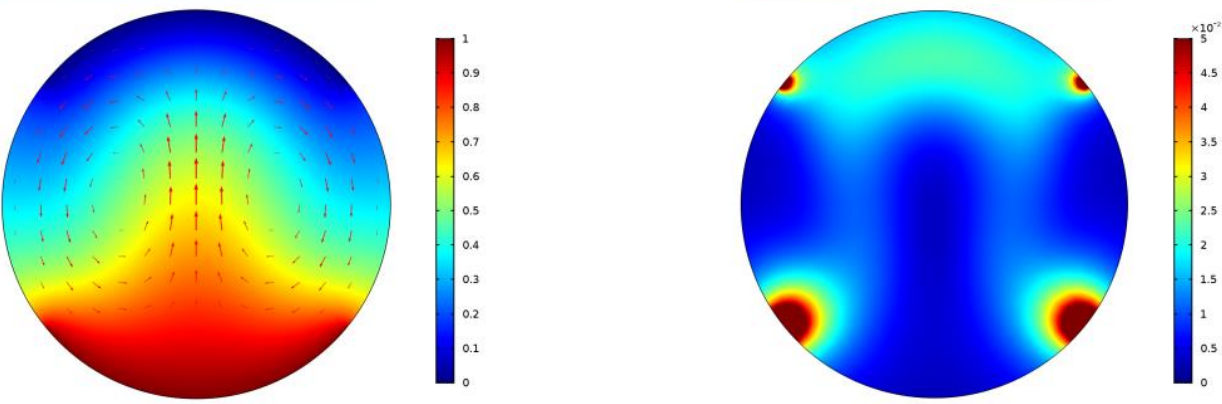

c
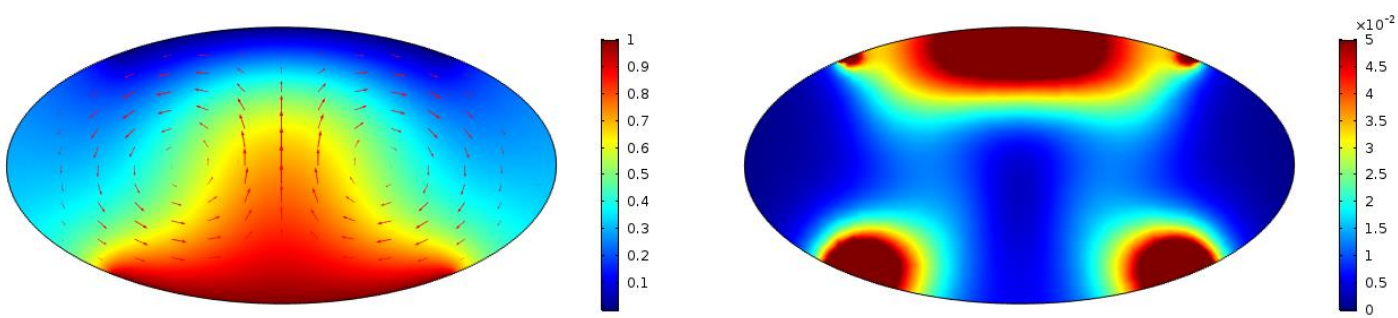

d
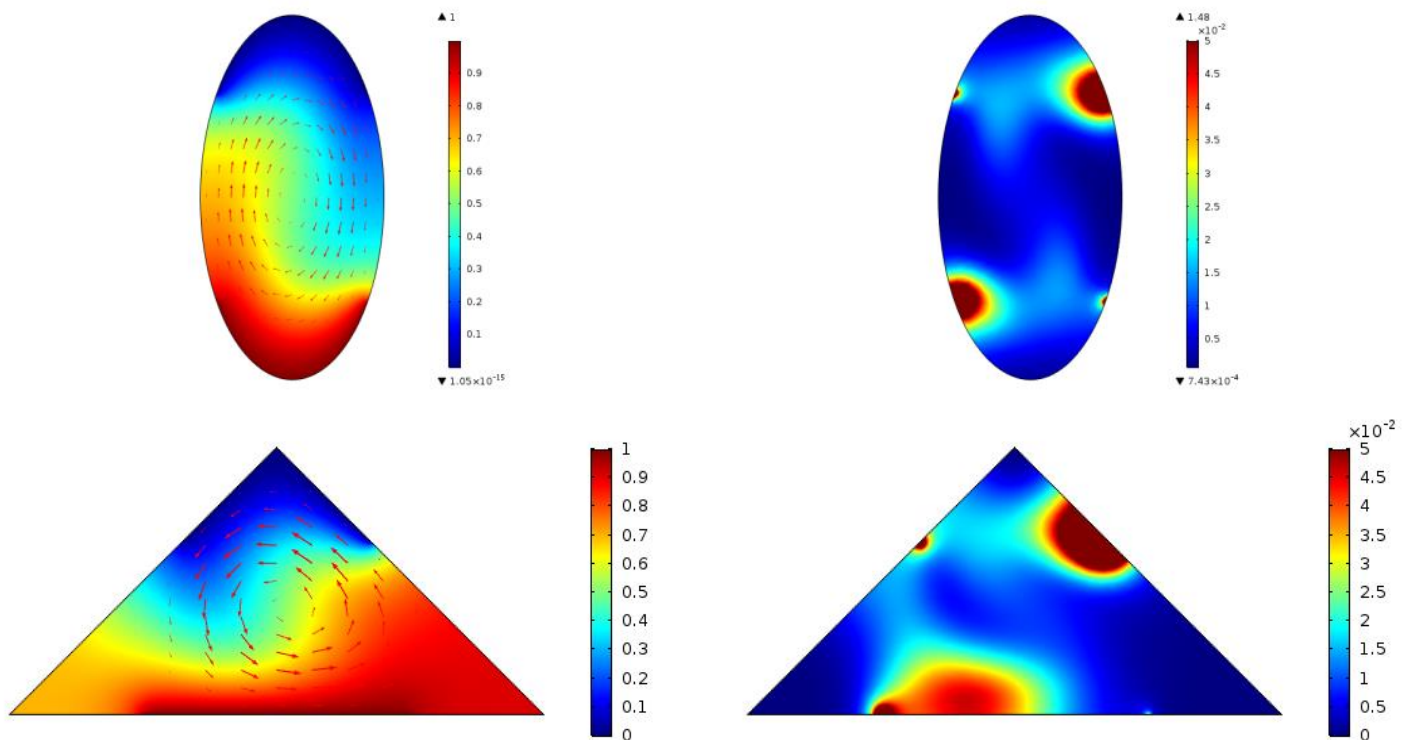

Figure 2. Temperature distribution with velocity vectors (left) and local entropy generation (right) for Gr $=1000$. 
temperature difference $\tau$ was set as 10 . This choice was made such that the temperature difference between the hot and cold walls was not too large.

To ensure the grid independency of the solution, the model was solved using different mesh sizes. For the two finest meshes, the total entropy generation $\widehat{\dot{S}}_{t}$ is given in Table 2. The results presented here are with a $G r$ of $1 \times 10^{3}$. The relative error in the prediction of $\hat{\dot{S}}_{t}$ was well below a relative error of $1 \%$.

In this section, results for the temperature, velocity vector fields, local and total entropy generation and Nusselt number at different Grashof numbers are presented. Figure 5 shows the temperatures distribution for the different geometries overlaid by the velocity vector field on the left side, and the local entropy generation on the right side. All the figures are for $G r$ of $1 \times 10^{3}$. For the square, circle and the horizontal ellipse, it can be noticed that the temperature distribution is symmetric about the center of the geometry. The buoyancy force resulting from this symmetrical temperature distribution, causes the fluid to rise from the center of the heated wall in a symmetric manner. This creates two circular motions on either sides of the geometries. In the case of the horizontal ellipse and triangle, the temperature distribution is not symmetric and hence, the flow domain is also not symmetric. From the different geometries studied, it is noticed that as the mean distance between the hot and cold boundaries decreases, the symmetric behavior can still be obtained at higher $G r$.

In Figure 5, the right column represents the local entropy generation for the different geometries. In Figure 5a, at a $G r$ of $1 \times 10^{3}$ the square configuration has the lowest local entropy generation. This is due to a uniform temperature distribution across the domain, unlike the other configurations, where a sharp temperature gradient exists at the endpoints of the hot and cold boundaries. Another observation is that as the hot and cold boundaries get closer to each other, a higher temperature gradient will be formed causing larger entropy generation in those areas as shown in Figure 5-c and e.

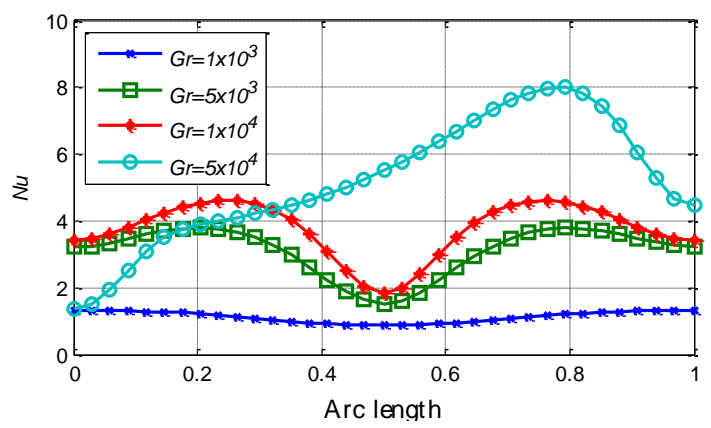

Figure 6. Nu on the hot wall for the square at different Gr.

$\mathrm{Nu}$ on the hot boundary for the square geometry is plotted in table 6 for different $G r$. This figure shows that for $G r \leq 1 \times 10^{4}$, the flow domain is symmetric and two eddies exist in the flow domain. These eddies then merge together at $G r=5 \times 10^{4}$ and the fluid starts to flow counterclockwise. At this $G r$, the highest $N u$ occurred around $\hat{x}=$ 0.8 and not at the end of the wall. This is due to the fact that the hot fluid continues to climb the right-side wall, lowering the rate of heat transfer in the $\hat{y}$ direction.

Figure 7 shows $\mathrm{Nu}$ on the hot wall for the circular geometry for different $G r$. Unlike the square, the symmetric pattern collapsed earlier and the solution is only symmetric for $G r=1 \times 10^{3}$. Another important distinction between the circular and square geometries is that the highest $\mathrm{Nu}$ is occurring at the beginning of the hot wall and not near its end. Since the flow is moving in the counter-clockwise direction, the highest temperature gradient is located at the point where the cold fluid touches the left hot side of the wall and hence increasing $\mathrm{Nu}$.

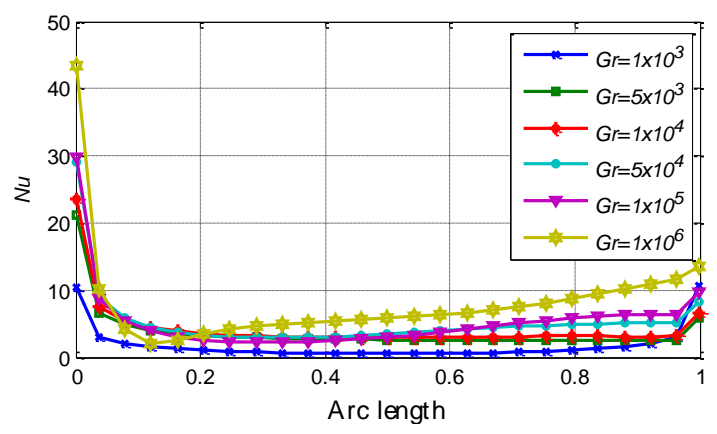

Figure 7. Nu on the hot wall for the circle at different Gr.

For the horizontal ellipse, the $\mathrm{Nu}$ is plotted in Figure 8 . The results obtained for this case are very similar to the circular shape. For these two configurations, the flow domain is not symmetric for $G r>1 \times 10^{3}$. Also, the highest $\mathrm{Nu}$ exists at the beginning of the hot wall for the same reason discussed in the case of the circle.

$\mathrm{Nu}$ on the hot boundary for the square geometry is plotted in Figure 6 for different $G r$. This figure shows that for $G r \leq 1 \times 10^{4}$, the flow domain is symmetric and two eddies exist in the flow domain. These eddies then merge together at $G r=5 \times 10^{4}$ and the fluid starts to flow counterclockwise. At this $G r$, the highest $N u$ occurred around $\hat{x}=$ 0.8 and not at the end of the wall. This is due to the fact that the hot fluid continues to climb the right-side wall, lowering the rate of heat transfer in the $\hat{y}$ direction.

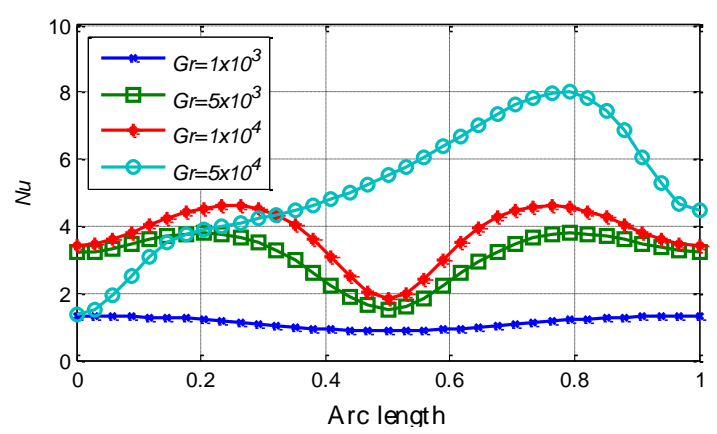

Figure 3. Nu on the hot wall for the square at different Gr.

Figure 7 shows $\mathrm{Nu}$ on the hot wall for the circular geometry for different $G r$. Unlike the square, the symmetric pattern collapsed earlier and the solution is only symmetric for $G r=1 \times 10^{3}$. Another important distinction between the circular and square geometries is that the highest $N u$ is occurring at the beginning of the hot wall and not near its end. Since the flow is moving in the counter-clockwise direction, the highest temperature gradient is located at the point where the cold fluid touches the left hot side of the wall and hence increasing $\mathrm{Nu}$.

For the horizontal ellipse, the $\mathrm{Nu}$ is plotted in Figure 5. The results obtained for this case are very similar to the circular shape. For these two configurations, the flow domain is not symmetric for $G r>1 \times 10^{3}$. Also, the highest 
$N u$ exists at the beginning of the hot wall for the same reason discussed in the case of the circle.



Figure 4. Nu on the hot wall for the circle at different $G r$.

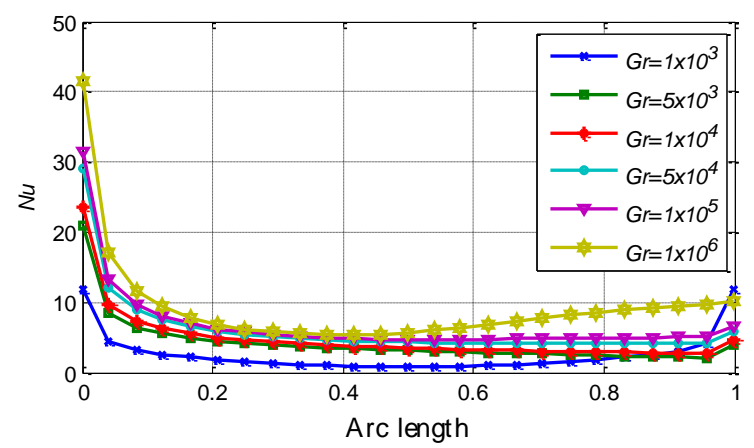

Figure 5. Nu on the hot wall for the horizontal ellipse at different $G r$.

Figure 6 shows $\mathrm{Nu}$ for the vertical ellipse. In this case $G r$ was increased only to $5 \times 10^{3}$, since no stable solution was obtained beyond this value. Unlike the previous case, even at $G r=1 \times 10^{3}$ the solution was not symmetric. $N u$ was higher on the right wall where the temperature gradient was higher. It can be noticed from this solution that increasing $\mathrm{Gr}$ does not always increase the $\mathrm{Nu}$, as shown in the case of $G r=5 \times 10^{3}$.

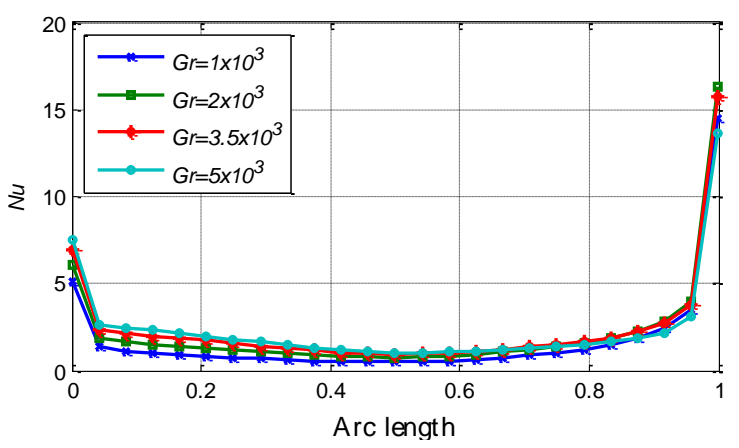

Figure 6. $\mathrm{Nu}$ on the hot wall for the vertical ellipse at different $G r$.

The local $\mathrm{Nu}$ for the triangle geometry is shown in Figure 7. Similar to the vertical ellipse, even at $G r=1 \times 10^{3}$ the solution was not symmetric and there was only one eddy in the flow domain. $N u$ was almost constant except on the left were the cold fluid was falling down and started to pass over the hot wall. In this configuration, $\mathrm{Nu}$ is directly related to $G r$ were it increases by increasing $G r$.

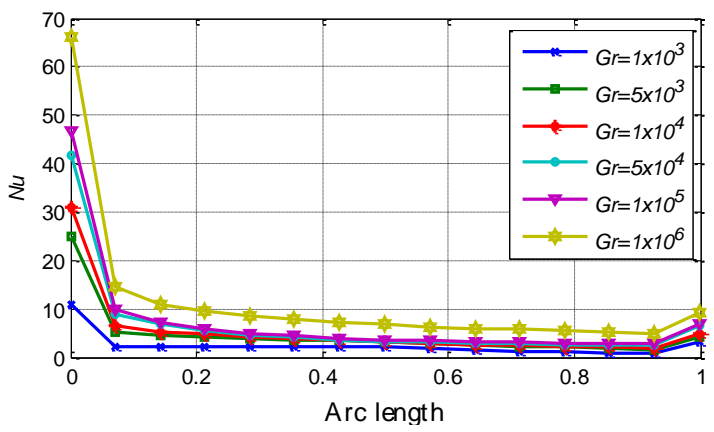

Figure 7. Nu on the hot wall for the triangle at different Gr.

$N u_{m}$ is plotted against $G r$ in Figure 8 for the different geometries. As anticipated, an increase in $G r$ provides more momentum to the eddies, and hence enhances the heat transfer rate, i.e., an increase in $N u$. The highest $N u$ for all $G r$ was obtained for the horizontal ellipse were the distance between the hot and the cold walls is the least. The square, horizontal ellipse and triangle geometries have relatively steeper monotonic behavior for $N u$ vs $G r$. It is noticed that as the mean distance between the hot and cold walls narrows, $N u_{m}$ increases. The circular shape behaves a bit different compared to the other geometries. For the circle, $N u_{m}$ tend to plateau around $G r=1 \times 10^{5}$. Around this specific $G r$ value, the characteristics of the flow domain change from being circular to flowing in an almost rectangular manner as will be further discussed in Figure 11. Beyond this, $N u$ continues to increase sharply with $G r$. The vertical ellipse on the other hand, has a moderate slope compared to the other geometries, due to the large distance between the hot and cold walls where the temperature gradient is the least.

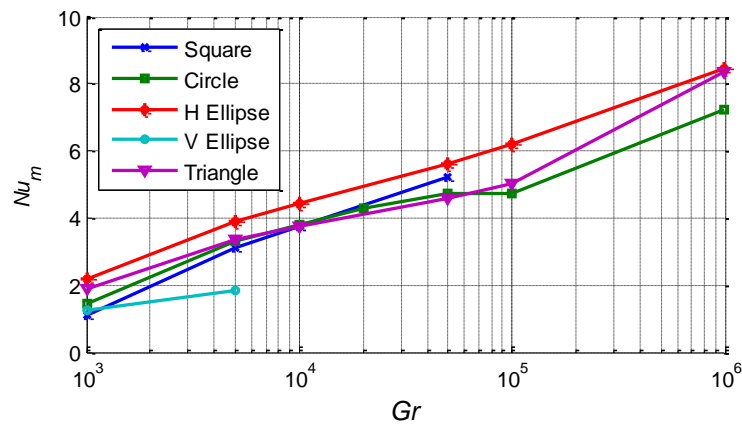

Figure 8. Mean Nu vs Gr for the different geometry.

Figure 9 shows the total entropy generation for the different geometries versus $G r$. In this study, the entropy generation that is due to viscous dissipation is very minimal. Thus, Bejan number $(B e)$ that is given by the ratio of the entropy generation due to heat transfer to the total entropy generation, i.e., the first term of Eq. (10) divided by the entire equation, is almost one. Therefore, it was decided not to report $B e$ in this study. Since the entropy generation is predominantly due to heat transfer, the entropy generation curves follow the heat transfer trend, i.e., $\mathrm{Nu}_{m}$. In this figure, again the highest entropy generation obtained was for the horizontal ellipse. For this shape the entropy generation is almost linear with $G r$. Generally as the hot and cold walls get closer to each other, the entropy generation tends to increase. 


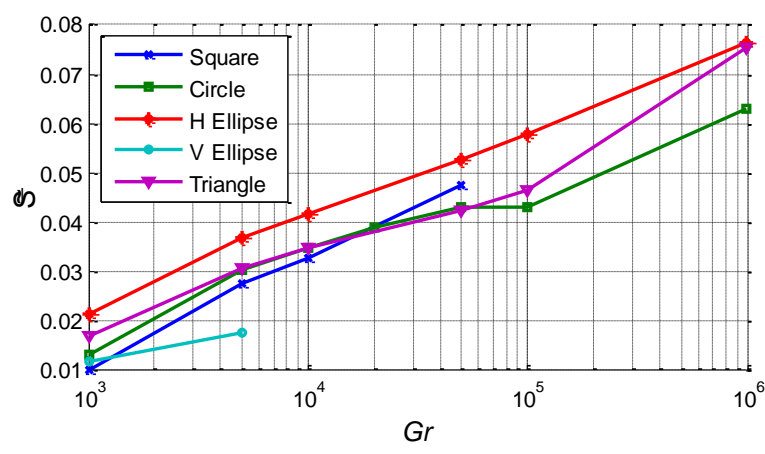

Figure 9. Total entropy generation vs Gr for the different geometries.

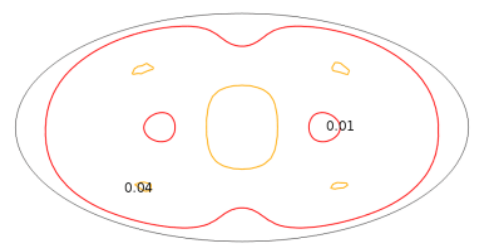

(a)

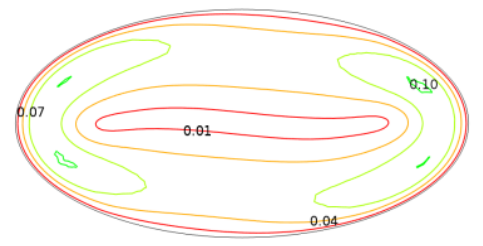

(c)
For the range $5 \times 10^{4} \leq G r \leq 1 \times 10^{5}$, the total entropy generation tends to increase at a significantly lower rate for the circular geometry in comparison to the other geometries. In order to analyze this phenomena, velocity contours are plotted for the horizontal ellipse, circular and triangular geometries in figures Figure 13-Figure 15, respectively. Figure 13 shows how the structure of the eddies is changing for the horizontal ellipse for $G r=1 \times 10^{3}$ to $G r=1 \times 10^{6}$. A noticeable change in the contour lines in the entire range of the $G r$ values shown.

For the circular geometry, only a very slight change in the eddies can be observed in the range of $5 \times 10^{4} \leq G r \leq$ $1 \times 10^{5}$ (see Figure $14 \mathrm{~b}$ and Figure $14 \mathrm{c}$ ), where the increase in the total entropy generation tends to slow down. Beyond this

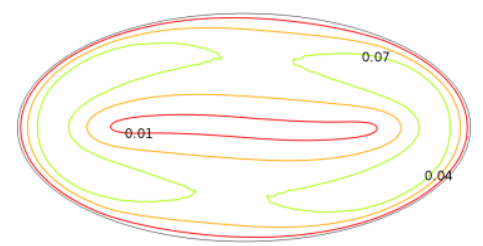

(b)

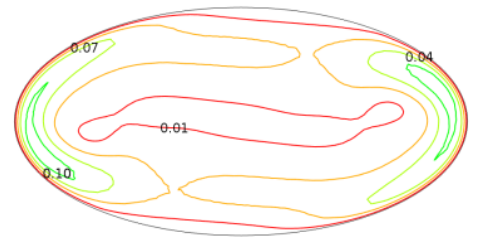

(d)

Figure 10. Velocity contours for horizontal ellipse at different $\mathrm{Gr} ;$ (a) $\mathrm{Gr}=1 \times 10^{3}$, (b) $\mathrm{Gr}=5 \times 10^{4},(\mathrm{c}) \mathrm{Gr}=1 \times 10^{5},(\mathrm{~d})$ $G r=1 \times 10^{6}$.

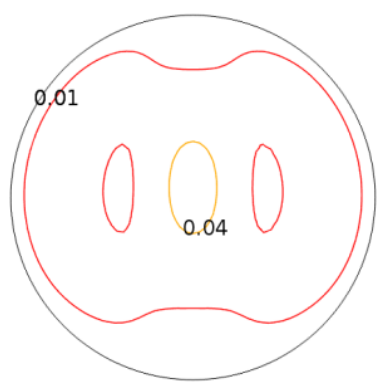

(a)

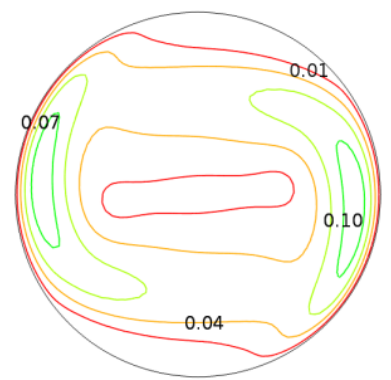

(c)

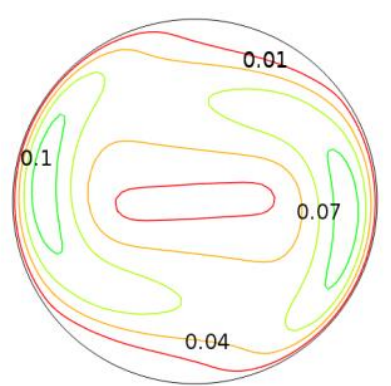

(b)

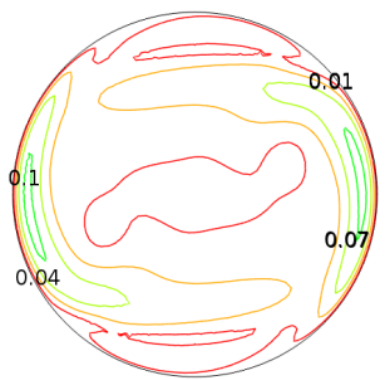

(d)

Figure 11. Velocity contours for circular shape at different $G r ;$ (a) $G r=1 \times 10^{3}$, (b) $G r=5 \times 10^{4},\left(\right.$ c) $G r=1 \times 10^{5},(d)$ $G r=1 \times 10^{6}$. 


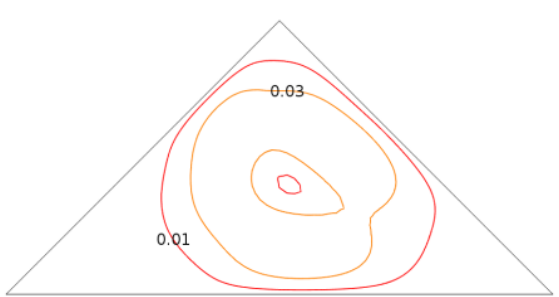

(a)

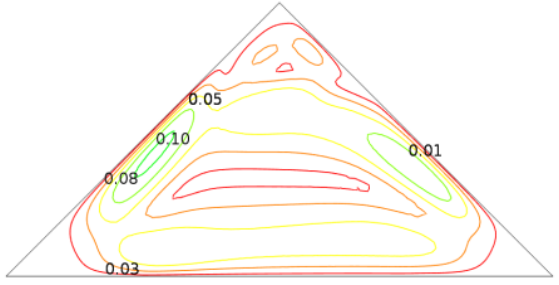

(c)

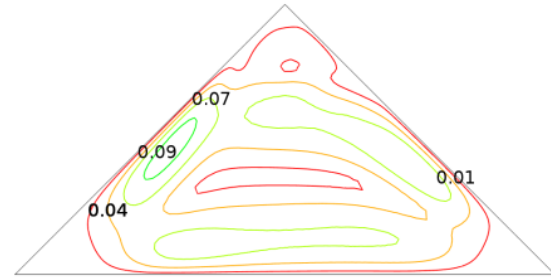

(b)

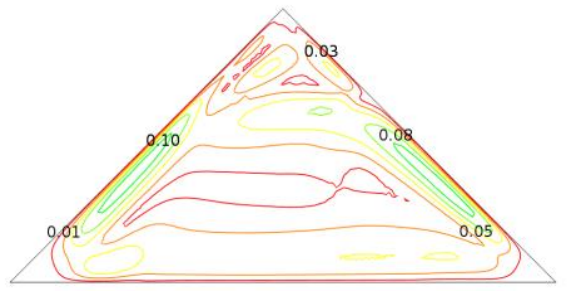

(d)

Figure 12. Velocity contours for triangular shape at different $\mathrm{Gr} ;$ (a) $\mathrm{Gr}=1 \times 10^{3}$, (b) $\mathrm{Gr}=5 \times 10^{4},(\mathrm{c}) \mathrm{Gr}=1 \times 10^{5},(\mathrm{~d})$ $G r=1 \times 10^{6}$

range eddies are highly distorted, which corresponds to a steeper increase in the total energy generation. Figure 15 shows that the velocity contours of the triangular geometry are significantly distorted, where several lines are being broken and new eddies are being formed in the flow domain. Thus, it can be concluded that the faster those eddies are distorted the higher the entropy generation rate will be.

\section{Conclusions}

Entropy generation for the natural convection phenomena was calculated using finite element method for different shapes that were subject to similar boundary conditions. Both the entropy generation and $\mathrm{Nu}$ were found to increase with an increase in the $\mathrm{Gr}$ in very similar trends. In this study, viscous dissipation had a minimal effect on the entropy generation, while the heat transfer was the dominant factor. The highest entropy generation was found to be for the horizontal ellipse geometry, while the vertical ellipse had the least entropy generation. The rate of entropy generation decreased as the distance between the hot and cold boundaries increased. The rate of increment for the entropy generation tended to decrease when the flow pattern started to change. This was more noticeable in the case of the circle and the triangle, where more eddies started to form when Gr was increased.

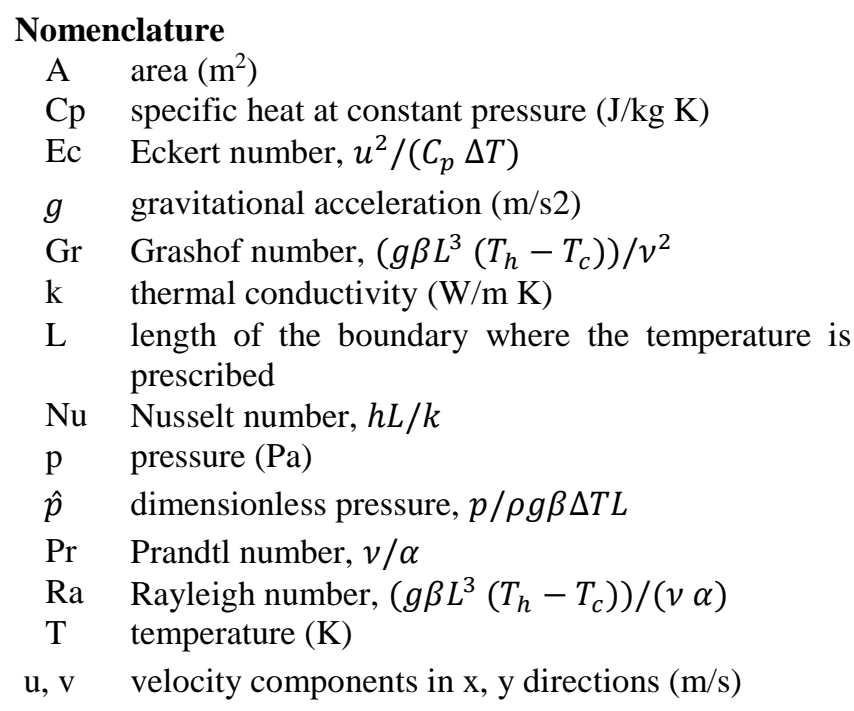

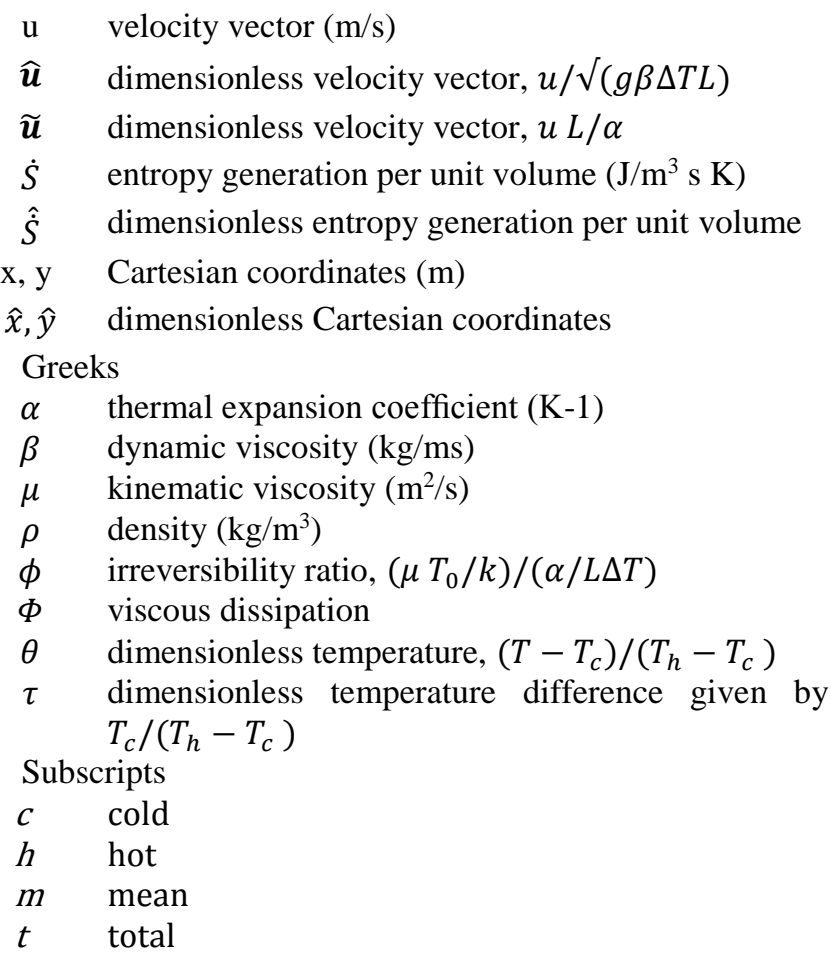

\section{References}

[1] M. Magherbi, H. Abbassi, A. Ben Brahim, "Entropy generation at the onset of natural convection," Int. J. Heat and Mass Transfer, 46, 3441-3450, 2003.

[2] P. A. K. Lam, K. Arul Prakash, "A numerical study on natural convection and entropy generation in a porous enclosure with heat sources," Int. J. Heat Mass Transfer, 69, 390-407, 2014.

[3] M. Famouri, K. Hooman, "Entropy generation for natural convection by heated partitions in a cavity," Int. Commun. Heat Mass Transfer, 35, 492-502, 2008.

[4] T. Basak, A. K. Singh, T. P. A. Sruthi, S. Roy, "Finite element simulations on heat flow visualization and entropy generation during natural convection in inclined square cavities," Int. Commun. Heat Mass Transfer, 51, 1-8, 2014. 
[5] A. C. Baytas, "Optimization in an Inclined Enclosure for Minimum Entropy Generation in Natura Convection," Journal of Non Equilibrium Thermodynamics, 22, 145-155, 1997.

[6] R. D. C. Oliveski, M. H. Macagnan, J. B. Copetti, "Entropy generation and natural convection in rectangular cavities," Applied Thermal Engineering, 29, 1417-1425, 2009.

[7] G. G. Ilis, M. Mobedi, B. Sunden, "Effect of aspect ratio on entropy generation in a rectangular cavity with differentially heated vertical walls," Int. Commun. Heat Mass Transfer, 35, 696-703, 2008.

[8] Y. Varol, H. F. Oztop, A. Koca, "Entropy production due to free convection in partially heated isosceles triangular enclosures," Applied Thermal Engineering, 28, 1502-1513, 2008.

[9] Y. Varol, H. F. Oztop, I. Pop, "Entropy generation due to natural convection in non-uniformly heated porous isosceles triangular enclosures at different positions," Int. J. Heat Mass Transfer, 52, 1193-1205, 2009.
[10] M. Abu-Qudais, E. A. Nada, "Entropy generation due to laminar natural convection from a horizontal isothermal cylinder," Journal Heat Transfer, 120, 10891090, 1998.

[11] B. A. K. Abu-Hijleh, W. N. Heilen, "Entropy generation due to laminar natural convection over a heated rotating cylinder," Int. J.Heat Mass Transfer, 42, 4225-4233, 1999.

[12] M. Aounallah, Y. Addad, S. Benhamadouche, O. Imine, L. Adjlout, D. Laurence, "Numerical investigation of turbulent natural convection in an inclined square cavity with a hot wavy wall," Int. J. Heat Mass Transfer, 50, 1683-1693, 2007.

[13] H. F. Oztop, K. Al-Salem, "A review on entropy generation in natural and mixed convection heat transfer for energy systems," Renewable Sustainable Energy Reviews, 16, 911-920, 2012.

[14] G. de Vahl Davis, I. Jones, "Natural convection in a square cavity: a comparison exercise," In. J. Numerical Methods Fluids, 3, 227-248, 1983. 